\title{
Effects of intestinal ischemia on in vitro activity of adjacent jejunum in samples obtained from ponies
}

\author{
Erin D. Malone, DVM, PhD, and Mathur S. Kannan, BVSc, PhD
}

\begin{abstract}
Objective-To determine whether intestinal ischemia would alter activity of the jejunum in vitro or alter staining characteristics for certain types of enteric neurotransmitters.
\end{abstract}

\section{Sample Population-Jejunal samples obtained from 10 ponies.}

Procedure-Jejunal samples were obtained from locations proximal and distal to an area of small intestine made ischemic for 60 minutes. A portion of each sample was stained to detect substance P-like immunoreactivity, cholinergic and adrenergic neurons, and nitric oxide synthase. Portions of the remaining samples were suspended in muscle baths. General activity patterns (frequency and amplitude of contraction), responses to neuronal depolarization induced by electrical field stimulation (EFS), and responses to $1 \mu \mathrm{M}$ norepinephrine (NE) were compared with responses of a normal section of small intestine obtained prior to ischemic insult.

Results-Staining patterns were not altered. Proximal and distal sections had evidence of decreased contractility, compared with the normal section. Contraction frequency also was decreased, and distal sections had lower contraction frequency than proximal sections. Relaxation responses were decreased in distal sections. Responses to NE differed significantly for distal and proximal sections, compared with normal sections.

Conclusions and Clinical Relevance-Short-term ischemia can significantly affect adjacent bowel. Contractile and relaxation responses are impaired. Discrepancies in intestinal motility patterns and alterations in response to NE for sections proximal and distal to ischemic intestine could lead to clinical ileus or slowed transit of ingesta. (Am J Vet Res 2001; 62:1973-1978)

$\mathbf{T}$ he success rate of surgeries for small intestinal lesions remains lower than that for large intestinal lesions, partly because of the increased risk of postoperative ileus (POI). ${ }^{1}$ It is believed that POI results from disruption in the balance between excitatory and inhibitory neurotransmitters secondary to inflammation, endotoxemia, surgical trauma, peritonitis, or intestinal distention. ${ }^{2}$

Received Jul 3, 2000

Accepted Dec 7, 2000.

From the Departments of Clinical and Population Sciences (Malone) and Veterinary PathoBiology (Kannan), College of Veterinary Medicine, University of Minnesota, St Paul, MN 55108.

Supported by the Minnesota Equine Research Center, College of Veterinary Medicine, University of Minnesota, and the Minnesota Racing Commission.

Presented at the 17th Annual Veterinary Medical Forum, Chicago, Ill, Jun 10-13, 1999, and the 9th Annual American College of Veterinary Surgeons Symposium, San Francisco, Calif, Oct 1-3, 1999.
Several therapeutic agents have been proposed for the treatment of horses with POI; however, many have undesirable adverse effects, and none are consistently effective. ${ }^{3}$ Additionally, drugs that are considered effective have varying mechanisms of action. Various types of lesions may have differing effects on intestinal function. Response to a particular drug likely depends on the type of lesion involved. Lack of understanding of the pathogenesis of POI makes it difficult to select an appropriate drug for a specific horse.

Several neurotransmitters function within the enteric nervous system of horses, including acetylcholine (Ach), norepinephrine (NE), nitric oxide (NO), and substance P (SP) or a related tachykinin. ${ }^{a}$ All 4 of these neurotransmitters have the potential to be involved in the pathogenesis of POI. The apparent effectiveness of drugs such as metoclopramide (enhances the release of Ach) and yohimbine $\left(\alpha_{2}\right.$-adrenoreceptor antagonist) suggests a role for altered release of Ach and NE. ${ }^{1,4}$ Substance P and NO have not been evaluated to determine whether they have a role in pathogenesis of POI; however, decreased amounts of SP have been documented early in the course of disease for horses with grass sickness, ${ }^{5}$ and both neurotransmitters are altered in some types of inflammatory bowel disease in humans. ${ }^{6,7}$ Additionally, NO modulates the hypotensive effects associated with lipopolysaccharides, and inhibitors of NO production can prevent changes in endotoxin-induced motility in rats. ${ }^{8,9}$

In people, bowel inflammation or injury substantially alters neuronal control mechanisms of the intestines, even after the initial lesion has resolved. ${ }^{10}$ Damaged small intestine usually is removed when possible, but it already may have altered motility control mechanisms of the remaining bowel. Alternately, minimally compromised bowel may be left in situ, but it may have alterations in release of neurotransmitters that could affect motility. We hypothesized that small intestinal ischemia in ponies would affect activity of bowel adjacent to the ischemic area. In particular, we hypothesized that changes in response to NE would be evident in bowel adjacent to ischemic intestine and that production of neurotransmitters such as SP and NE would be altered in adjacent intestine. A combination of in vitro muscle responses and immunohistochemical analysis was chosen to investigate such changes following short-duration intestinal ischemia in ponies.

\section{Materials and Methods}

Sample collection-Ten ponies anesthetized for a teaching surgery laboratory at our facility were used in the study. 
For each pony, the mid-jejunum was identified at the start of surgery, and a $30-\mathrm{cm}$ section was isolated, using Penrose drains to prevent leakage of intestinal contents. A central 2.5$\mathrm{cm}$ section of this isolated segment was removed and served as the normal-control sample. Vessels supplying the remainder of the isolated segment were transected, and mesenteric vasculature from adjacent bowel was ligated. After 60 minutes, a period sufficient to induce substantial damage to the lamina propria and villi, ${ }^{11}$ samples $(2.5 \mathrm{~cm})$ were obtained from the jejunum $2.5 \mathrm{~cm}$ proximal and $2.5 \mathrm{~cm}$ distal to the devascularized bowel. A sample of devascularized bowel also was obtained. For each tissue sample, half was placed in neutral-buffered $10 \%$ formalin. The other half was incised along the mesenteric border, and the sample was opened and placed in oxygenated Krebs bicarbonate buffer solution.

Measurement of muscle activity-All samples were tested within 12 hours after collection. Normal, proximal, and distal sections from 9 horses were evaluated. The mucosa and submucosa were removed by sharp dissection, and the remaining tissue was cut into $2 \times 10$-mm strips oriented in a longitudinal or circular plane. Strips were suspended isometrically under a tension of 2 to $3 \mathrm{~g}$ (determined by optimum contraction amplitude) in organ baths containing oxygenated Krebs bicarbonate buffer maintained at 38.5 C. Changes in tension were recorded, using a force displacement transducer connected to a polygraph. Tissues were allowed to equilibrate for 1 hour. Maximum contractility was determined by use of $90 \mathrm{mM} \mathrm{KCl}$. Tissues were washed and allowed to recover for 10 minutes between treatments. At the end of testing, tissues were washed, and the response to $\mathrm{KCl}$ was repeated to assess muscle fatigue. Paired $t$-tests were used to determine significant changes in the response to $\mathrm{KCl}$. Responses to $\mathrm{KCl}$ were adjusted among strips, using crosssectional area of the muscle. ${ }^{12}$

Initial muscle activity after the 1-hour equilibration period was analyzed on the basis of peak amplitude, frequency of contraction, and area under the curve for a l-minute period. Peak amplitude and area under the curve were adjusted on the basis of mean response to $\mathrm{KCl}$ for that strip. Values for normal, proximal, and distal sections were compared, using repeated-measures ANOVA.

Electrical field stimulation (EFS) was performed at maximal (30 V for $4 \mathrm{~Hz}$ ) and submaximal $(20 \mathrm{~V}$ for $2 \mathrm{~Hz}$ ) levels and at 1-millisecond pulses for a 10-second train to stimulate neurotransmitter release. ${ }^{\text {a }}$ Studies were conducted at low tension (ie, baseline tension) before and after administration of $1 \mu \mathrm{M}$ NE and also while tissues were at high tension following the final $\mathrm{KCl}$ treatment. Response to EFS at low tension was evaluated on the basis of peak at the end of EFS (termed an "on" response) and 6 seconds (approximate peak response) following EFS (an "off" response). Data were adjusted on the basis of mean responses to $\mathrm{KCl}$ for that strip. Response to EFS at high tension was evaluated as a percentage of the initial tension at the end of EFS (ie, on response) and 6 seconds following EFS (ie, off response). Values for normal, proximal, and distal sections were compared, using repeated-measures ANOVA.

Norepinephrine response-To determine whether increased concentrations of circulating catecholamines could affect bowel motility, longitudinal and circular muscle strips of normal, proximal, and distal sections for each pony were tested for response to $1 \mu \mathrm{M}$ NE. The 3 sections from each pony were tested as a unit to prevent variation attributable to time of experiment. Only 1 test was conducted on each strip because of tachyphylaxis associated with NE. In another study $^{13}$ on equine jejunum, longitudinal muscle exposed to NE initially had increases in contraction amplitude and tone followed by decreases in tone as well as contraction ampli- tude and frequency. Circular muscle strips did not have an initial increase in activity but did have decreases in tone and activity following NE administration. Therefore, analysis of any initial contractile response in longitudinal muscle was performed on the basis of peak contraction amplitude, area under the curve for the first minute after NE administration, and area under the curve for the duration of contractile response (time until tone returned to baseline values). Analysis of the delayed relaxant response in longitudinal muscle and the response in circular muscle was performed in terms of peak contraction amplitude for a 1-minute period evaluated beginning 15 seconds after NE administration, tone of the plateau, and frequency of contraction for 1 minute after tone returned to baseline values, compared with a 1-minute period prior to NE administration. Comparisons were made between proximal, distal, and normal sections in terms of contraction amplitudes, area under the curve, duration responses, contraction frequency, and muscle tone, using repeated-measures ANOVA. Effects of NE on the response to EFS also were evaluated. Changes in EFSinduced responses of a strip before and after addition of $1 \mu \mathrm{M}$ $\mathrm{NE}$ were calculated, and differences between normal, proximal, and distal sections were compared, using repeated-measures ANOVA.

Histochemical analysis-To prevent shrinkage, sections were pinned flat in a silicone-coated dish. ${ }^{\mathrm{b}}$ After fixation in neutral-buffered $10 \%$ formalin for 2 hours, sections were washed with PBS solution and placed into PBS solution supplemented with 20\% sucrose for cryopreservation. Sections subsequently were frozen in embedding media at $-70 \mathrm{C}$, cut on a cryostat $(-25 \mathrm{C})$ at a thickness of $15 \mu \mathrm{m}$, and placed on gel-coated slides. Six sections of each tissue ( 3 oriented along fibers of the circular muscle and 3 oriented along fibers of the longitudinal muscle) were placed on each slide. For each of 10 ponies, samples of normal, proximal, and distal sections as well as a sample of devitalized bowel were examined.

Immunohistochemical analysis-Slides were washed in $10 \%$ casein for 1 hour and rinsed twice with PBS solution. Tissues were absorbed with $10 \%$ serum to reduce background staining and then stained to detect SP or choline acetyltransferase (Chat), which served as a marker for Ach. Slides were incubated with the primary antibody (rabbitderived antibody to Chat [dilution, 1:2,000] or rabbitderived antibody to SP [dilution, 1:800]) for 48 hours at 4 C. Goat-anti-rabbit (GAR)-Cy3 (dilution, 1:50) was used as fluorescence-labeled secondary antibody. Slides were incubated with GAR-Cy3 for 1 hour in the dark. Slides then were washed 4 times and allowed to dry; cover slips were then applied. Separate slides were stained in a similar manner to detect dopamine- $\beta$-hydroxylase (DBH) activity, which served as a marker for noradrenergic neurons, using sheepderived antibody to DBH and a fluorescein isothiocyanate (FITC)-labeled secondary antibody (dilution, 1:50). Optimum dilution of DBH antibody was 1:800 for a doublelabeling protocol. Specificity was tested for SP staining by absorbing the antibody with $15 \mu \mathrm{g}$ of SP/ml prior to staining; similarly, specificity for DBH staining was tested by absorbing the antibody with $25 \mu \mathrm{g}$ of $\mathrm{DBH} / \mathrm{ml}$ prior to staining. Equine spinal cord was used as a positive-control sample. Serum was used for negative-control samples. Slides were evaluated within 72 hours, using fluorescence and confocal microscopes.

Slides from each of the 3 sections of the jejunum were evaluated to detect the reduced form of nicotinamide adenine dinucleotide phosphate-diaphorase (NADPH-d) activity by incubating slides with a solution containing $\beta$-NADPH and nitroblue tetrazolium. These compounds react with NADPH-d in neurons containing NO synthase and form for- 
mazan deposits in the cytoplasm. Solution without nitroblue tetrazolium was used for negative-control samples. Slides were incubated at $30 \mathrm{C}$ and evaluated between 1 and 2 hours after initiation of incubation.

The DBH and NADPH-d slides also were stained to detect c-fos by use of the aforementioned protocol for immunohistochemical analysis, using rabbit-derived antibody to c-fos (dilution, 1:1,000) and GAR-Cy3 (dilution, $1: 200$ ). Equine spinal cord served as a positive-control sample. Specificity was tested by absorbing the antibody with a blocking peptide prior to staining.

Number of neuronal cell bodies that had positive staining was counted in 3 sections for each sample. In the case of DBH and NADPH-d slides, neurons had to stain for c-fos and the specific enzyme (DBH or NAPDH-d) to document enzymatic activity. ${ }^{14}$

All slides then were stained with H\&E. The number of neuronal cell bodies in each of the sections was counted, and sections of ischemic bowel were assessed to determine the amount of damage. Percentage of neuronal cell bodies that stained positively was compared among tissue sections, using an ANOVA and the least significant difference test. Photographs were taken of all sections to allow subjective comparisons for additional observers, if needed.

Statistical analysis-Significance for all tests was designated at $P<0.05$. When significant differences were detected by use of the repeated-measures ANOVA, a least significant difference test or Duncan test for multiple comparisons was used to detect differences among groups.

\section{Results}

Muscle activity-Peak response to $\mathrm{KCl}$ did not change from the beginning to the end of the procedure for each strip. This indicated that muscle strips were not fatigued and still could respond maximally.

Compared with normal sections, baseline activity of proximal and distal sections was altered for both longitudinal and circular muscle. For longitudinal muscle strips, the frequency of contraction was decreased in proximal and distal sections, and distal sections had a significantly lower contraction frequency than proximal sections (Fig 1). Distal sections

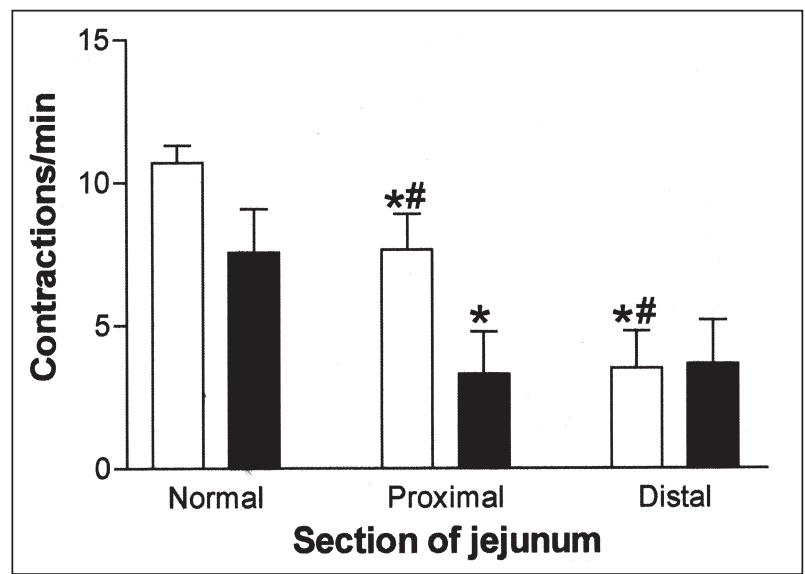

Figure 1-Alterations in baseline contractile frequency (mean \pm SEM) in sections of jejunum of 9 ponies. Results for longitudinal muscle (white bar) and circular muscle (black bar) were determined in a normal section of jejunum obtained before induction of ischemia and sections obtained after exposure to ischemic conditions from locations proximal and distal to the ischemic area. ${ }^{*}$ Value differs significantly $(P \leq 0.05)$ from value for normal section. \#Values with the same symbol differ significantly ( $P \leq$ 0.05). also had significantly decreased peak responses, compared with normal sections (Fig 2). Peak response to $\mathrm{KCl}$ (adjusted for cross-sectional area) also was significantly $(P=0.01)$ lower for longitudinal muscle strips of proximal (mean \pm SEM, $1.2 \pm 0.2 \mathrm{~g} / \mathrm{mm}^{2}$ ) and distal $\left(1.1 \pm 0.2 \mathrm{~g} / \mathrm{mm}^{2}\right)$ sections, compared with longitudinal muscle strips of normal sections (1.9 \pm $0.1 \mathrm{~g} / \mathrm{mm}^{2}$ ).

Circular muscle strips in proximal sections also had significantly decreased frequency of contraction, compared with frequency of contraction for normal sections (Fig 1). A similar pattern was evident for distal sections; however, those values did not differ significantly $(P=0.08)$. Area under the curve for peak response to $\mathrm{KCl}$ was significantly $(P=0.03)$ decreased for proximal $\left(0.55 \pm 0.4 \mathrm{~mm}^{2}\right)$ and distal $\left(0.58 \pm 0.4 \mathrm{~mm}^{2}\right)$ sections, compared with values for the normal sections $\left(1.87 \pm 0.4 \mathrm{~mm}^{2}\right)$. Peak amplitudes were not significantly different among the 3 groups (Fig 2).

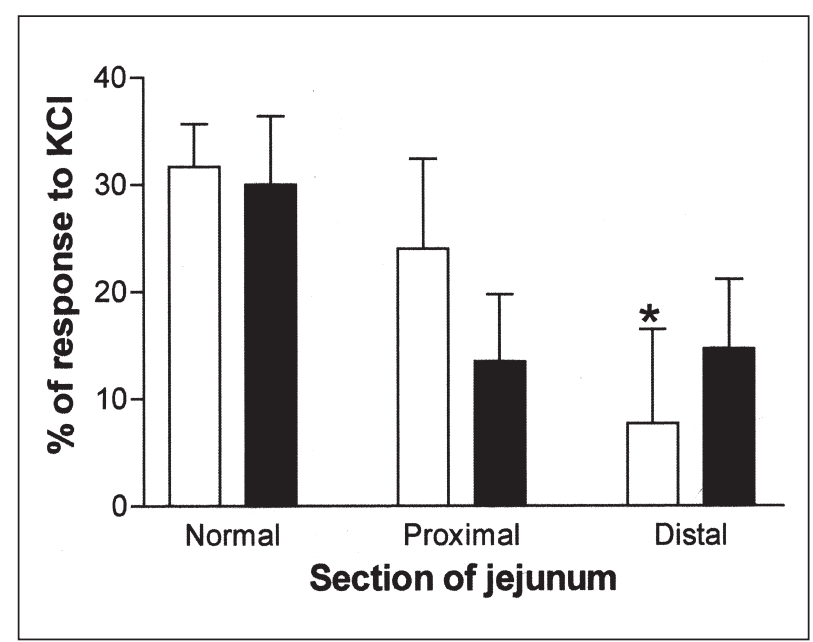

Figure 2-Alterations in baseline peak contraction amplitude (mean \pm SEM) in sections of jejunum of 9 ponies. See Figure 1 for key.

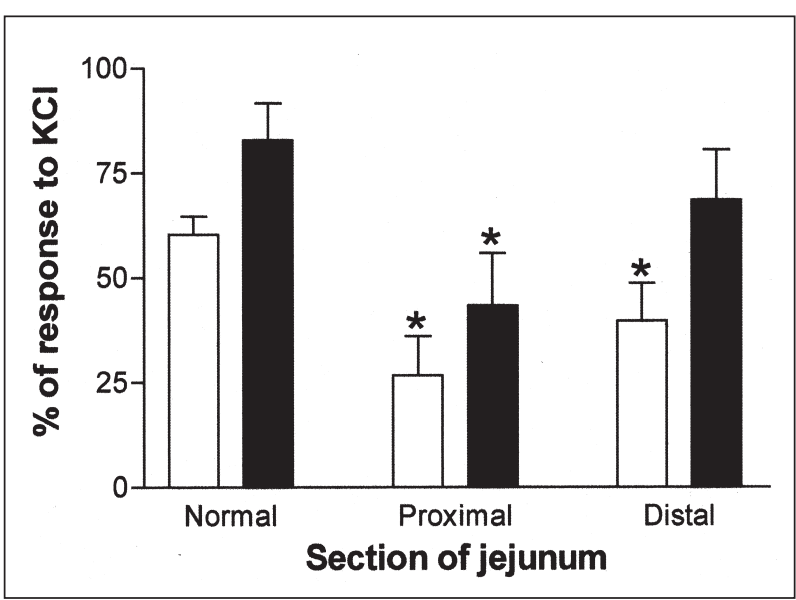

Figure 3-Alterations in the electrical field stimulation (EFS)induced response (mean \pm SEM) of longitudinal muscle strips under low tension in sections of jejunum of 9 ponies. Responses reported are on the basis of peak at the end of EFS (20 V, white bar; $30 \mathrm{~V}$, black bar). See Figure 1 for remainder of key. 
Response to EFS-induced neuronal depolarization-When longitudinal muscle strips under low tension were exposed to EFS of $20 \mathrm{~V}$ to stimulate neuronal depolarization, the on response (ie, during stimulation) was significantly greater in amplitude for muscle in normal sections, compared with muscle in proximal or distal sections (Fig 3). At EFS of $30 \mathrm{~V}$, the on response of normal sections was significantly greater than that of proximal sections but not greater than that of distal sections. Significant differences were not detected for the off response (ie, response immediately following EFS) at either stimulation level (for EFS of $30 \mathrm{~V}$, results [as a percentage of response to $\mathrm{KCl}$ ] were as follows: normal, $22.3 \pm 5.0 \%$; proximal, $23.6 \pm$ $7.0 \%$; distal, $18.7 \pm 6.7 \%$ ). In longitudinal muscle strips under high tension, a significant difference could not be detected among groups at EFS of $20 \mathrm{~V}$ for $2 \mathrm{~Hz}$ (data not shown); however, at EFS of $30 \mathrm{~V}$ for $4 \mathrm{~Hz}$, normal and proximal sections had significantly more relaxation during and after EFS than distal sections (Fig 4). Under these conditions, normal and proximal

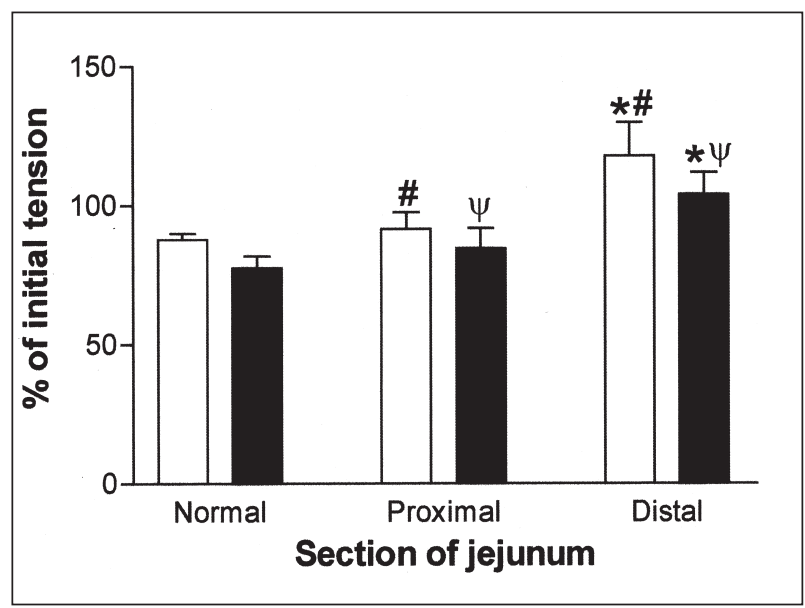

Figure 4-Alterations in the EFS-induced response (mean \pm SEM) of longitudinal muscle strips under high tension (contracted by $\mathrm{KCl}$ ) in sections of jejunum of 9 ponies. Values reported are on the basis of peak response at the end of EFS of $30 \mathrm{~V}$ (white bar) and response obtained approximately 6 seconds after EFS of $30 \mathrm{~V}$ (black bar). $\psi$ Values with the same symbol differ significantly $(\mathrm{P} \leq 0.05)$. See Figure 1 for remainder of key.

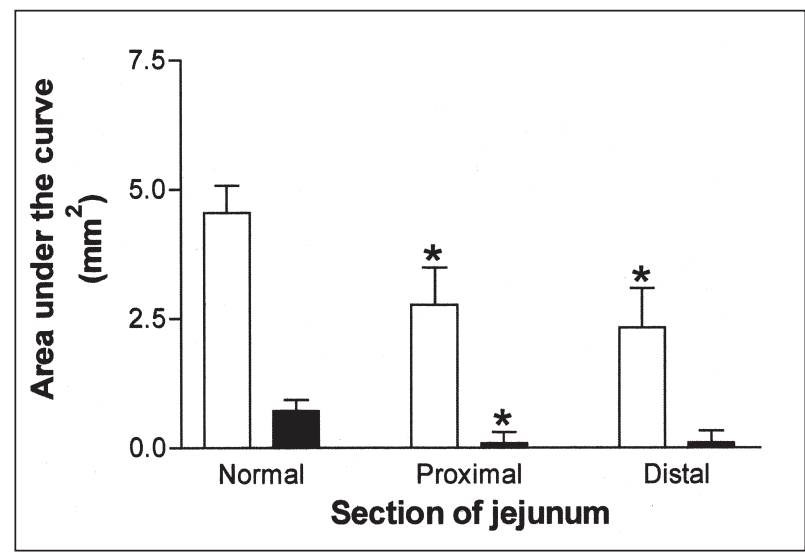

Figure 5-Alterations in the initial response (mean \pm SEM) for longitudinal muscle (white bar) and circular muscle (black bar) after addition of $1 \mu \mathrm{M}$ norepinephrine (NE) in sections of jejunum of 9 ponies. See Figure 1 for key. sections relaxed, compared with the initial tension, whereas distal sections contracted.

Differences were not detected among groups for circular muscle strips for either set of stimulation levels when under low or high tension (data not shown).

Response to NE-In longitudinal muscle strips exposed to $1 \mu \mathrm{M} \mathrm{NE}$, distal sections had significantly lower values, compared with normal sections, for initial contraction amplitude (35.1 $\pm 9.4 \%$ of response to $\mathrm{KCl}$ vs $61.0 \pm 6.5 \%$ of response to $\mathrm{KCl} ; P=0.03)$, tone $(17.4$ $\pm 7.0 \%$ of $\mathrm{KCl}$ response vs $43.3 \pm 4.8 \%$ of response to $\mathrm{KCl} ; P=0.01)$, and area under the curve $(2.33 \pm 0.76$ $\mathrm{mm}^{2}$ vs $4.55 \pm 0.53 \mathrm{~mm}^{2}$; Fig 5 ). Distal sections also had increased tone during the relaxation phase, compared with normal sections (Fig 6). A similar pattern was detectable in proximal sections, but those values did not differ significantly $(P=0.07)$ from values for normal sections. When longitudinal muscle strips under low tension were stimulated with EFS after the

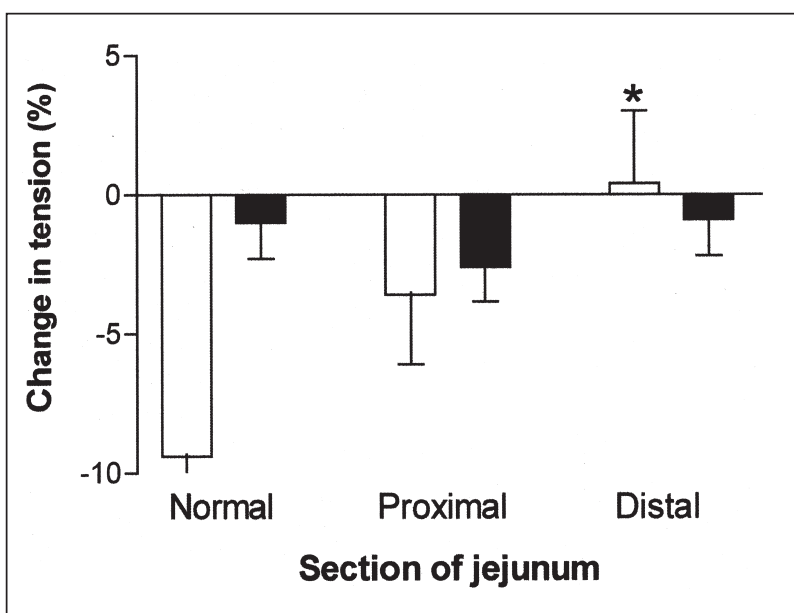

Figure 6-Alterations in the secondary relaxation response (mean \pm SEM) for longitudinal muscle (white bar) and circular muscle (black bar) after addition of $1 \mu \mathrm{M} N \mathrm{NE}$ in sections of jejunum of 9 ponies. Change in tension represents percentage of maximal response to KCl. See Figure 1 for key.

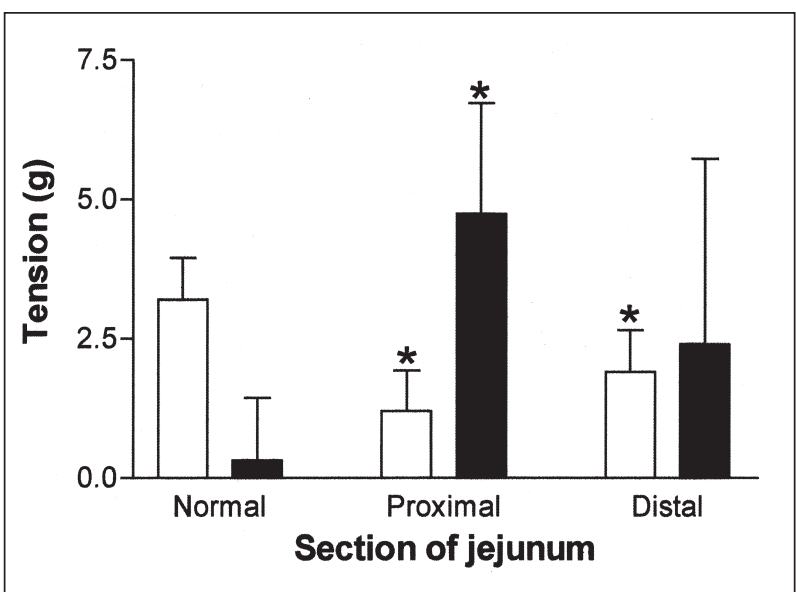

Figure 7-Alterations in jejunal response to EFS (mean \pm SEM) for longitudinal muscle (white bar) and circular muscle (black bar) on the basis of peak response at the end of EFS after addition of $1 \mu \mathrm{MNE}$ to muscle strips under low tension. See Figure 1 for key. 
addition of NE, normal sections had an increase in contractile response during stimulation, compared with the response without NE. This increased response was not observed in proximal or distal sections (Fig 7).

In circular muscle strips, distal sections had significantly decreased contraction amplitude after addition of $\mathrm{NE}(1.8 \pm 2.9 \%$ of response to $\mathrm{KCL})$, compared with normal sections $(10 \pm 2.9 \%$ of response to $\mathrm{KCl}$ ). Proximal sections had a lower area under the curve than normal sections (Fig 5), and a similar but not significantly different pattern was apparent in distal sections. Duration of the relaxant response to NE also was significantly prolonged in proximal sections (Fig 8). In general, circular muscle strips under low tension did not have a substantial contractile on response to EFS; however, after addition of NE, circular muscle strips under low tension in proximal sections contracted strongly. This response was significantly greater than that for normal sections (Fig 7).

Histologic examination-Ischemic tissues had loss of epithelium and blunting of villi with minor amounts of reepithelialization. This was not detected in samples from adjacent bowel or control samples. We did not detect inflammatory cell infiltrate in any of the samples.

Positive results were obtained for all immunohistochemical stains, compared with results of positiveand negative-control samples. However, staining for cfos-like immunoreactivity was minimal in all sections. Subsequently, alterations in NADPH-d or DBH staining were not evaluated, because we were interested in activity of these enzymes instead of only the detection of adrenergic or NO synthase-containing neurons.

Substance P-like immunoreactivity was noticeable in all sections (mean \pm SD, $46.4 \pm 14 \%$ of myenteric plexus neurons) and was similar in distribution and amount to that reported for clinically normal horses. ${ }^{15}$ Significant differences were not detected among ischemic bowel or normal, proximal, and distal sections. Choline acetyltransferase-like immunoreactivity was detected in low amounts (3.3 $\pm 1.2 \%$ of myenteric plexus neurons). Significant differences were not detected among ischemic bowel or normal, proximal, and distal sections.

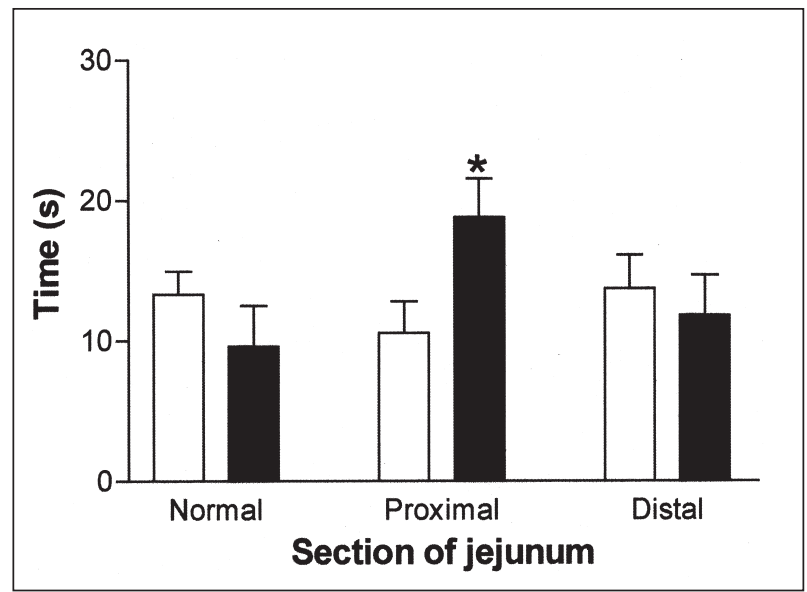

Figure 8-Alterations in duration of relaxation (mean \pm SEM) for longitudinal muscle (white bar) and circular muscle (black bar) after addition of $1 \mu \mathrm{MNE}$ in sections of jejunum of 10 ponies. See Figure 1 for key.

\section{Discussion}

Analysis of results of the in vitro portion of the study reported here suggests that short-duration ischemia causes alterations in function of adjacent bowel. Changes were detected in the general muscle response, response to NE, and response to EFS (general neuronal stimulation). However, because of a lack of alterations in staining patterns, these changes likely reflect alterations in the muscle's ability to contract or changes in receptor number and function rather than production or release of the tested neurotransmitters.

Longitudinal muscle adjacent to an ischemic lesion was not capable of contracting as strongly as normal bowel, as determined on the basis of baseline activity (Fig 2), response to $\mathrm{KCl}$, or response to neuronal depolarization (Fig 3). This difference was particularly evident in proximal sections. Propulsive movement of ingesta requires coordination between enteric afferent and efferent neurons to produce narrowing of the gastrointestinal tract orad to a bolus and widening aborally. ${ }^{16,17}$ Because contraction of longitudinal muscle widens the lumen, a weaker contractile response could hinder passage of ingesta proximal to an ischemic site. Circular muscle was affected less substantially but did have evidence of decreased strength and duration of contractions. Both muscle layers also had altered contraction frequency after ischemia (Fig 1). Altered contraction frequency would have the potential to further affect propulsive activity or segmentation. ${ }^{16}$

Changes in contractile response may reflect alterations in muscle function, neurotransmitter release, or hormonal influences. Ischemia may affect bowel directly (hypoxia) or indirectly by induction of inflammation or pain. Inflammation and pain may cause local and systemic effects. Because the ponies were anesthetized for the duration of the study, pain was unlikely to be an influence in this experiment. Interleukins and prostaglandins have effects on motility and enteric muscle function, ${ }^{18,19}$ and studies in rats suggest bowel manipulation stimulates an inflammatory response that has direct effects on activity of circular muscle. ${ }^{20}$ We cannot totally exclude effects attributable to an additional hour of anesthesia or additional bowel manipulation, but ponies were maintained at a stable plane of anesthesia at the start of the experiment, bowel manipulation was avoided as much as possible once samples were obtained, and we were unable to detect evidence of inflammation histologically. We could not find evidence for altered neurotransmitter release; amount of SP and Chat appeared unchanged after short-duration ischemia. We also did not detect evidence of induction of neuronal activity characterized by c-fos values. The c-fos is an immediate early gene and was used to determine whether neurons containing DBH and NADPH-d had altered function, compared with control samples, because staining for the enzyme itself would indicate detection of the enzymes but not enzyme activity. ${ }^{14}$ Because we did not detect changes in C-fos values, we concluded these enzymes did not have altered activity after short-duration ischemia. However, the function of neurotransmitters may still be altered by changes in the muscle's ability to respond (as indicat- 
ed by the altered response to $\mathrm{KCl}$ ) or by changes in number or function of receptors.

Longitudinal muscle in distal sections relaxed less in response to neuronal depolarization, compared with relaxation in normal sections. In fact, when under higher tension, it appeared to contract above baseline values (Fig 4). These changes, particularly when disparate among anastomotic sections, could hinder motility. Potentially, this change in function could reflect denervation changes distal to an ischemic lesion, changes in type of neurotransmitters released, or alterations in receptor-mediated functions. Changes in activity of the sodium-potassium pump reportedly produce a similar decrease in smooth muscle relaxation (leading to overall increased contraction) in nematode-infected rats. ${ }^{21,22}$

When muscle strips were exposed to NE, differences were apparent between normal sections and proximal or distal sections. Longitudinal and circular muscle strips had evidence of weaker contractile responses (Figs 5 and 8). Hypoxia decreased the amount of tension generated in response to NE in pulmonary artery rings obtained from horses in another study. ${ }^{c}$ These types of changes could reflect alterations in the muscle's ability to respond or in altered muscle function as a result of binding to $\alpha_{2^{-}}$or $\beta$-adrenoceptors. Desensitization or tachyphylaxis also could develop secondarily to increased concentrations of circulating catecholamines. ${ }^{23}$ However, the latter would not account for the discrepancy between proximal and distal sections or for the longer duration of relaxation in circular muscle strips. On the other hand, catecholamines can have a presynaptic inhibitory effect on cholinergic and peptidergic transmission and also could affect contractile responses via these pathways. ${ }^{24,25}$

Under certain conditions, muscle responses were characterized by significantly decreased relaxation. After addition of $\mathrm{NE}$, longitudinal muscle strips of proximal and distal sections did not relax as much as muscle strips of normal sections (Fig 6), and circular muscle strips contracted strongly in response to EFS instead of relaxing (Fig 7). These responses could reflect a lesion involving enteric inhibitory neurons (ie, unmodulated contraction), ${ }^{16}$ muscle function, ${ }^{23}$ or altered $\beta$-adrenoceptor-mediated function. ${ }^{24}$

Clinical importance of these changes is difficult to predict; however, the response patterns do suggest that treatment emphasis should be placed on restoring function of longitudinal muscles and minimizing the release of NE and epinephrine, as has been suggested by other investigators. ${ }^{4}$ Ability to determine the most likely form of motility dysfunction of the small intestines that follows intestinal resection and anastomosis for removal of ischemic lesions will aid in the selection of the most appropriate motility modifiers and decrease the duration of hospitalization, costs of treatment, and fatality rates currently associated with POI. Additionally, insight into the pathogenesis of POI may allow for development of new drugs or drug combinations that are more effective in the treatment of horses with POI.

\footnotetext{
${ }^{a}$ Malone ED. Neural control of equine intestinal motility. PhD thesis,

Department of Clinical and Population Sciences, College of

Veterinary Medicine, University of Minnesota, St Paul, Minn, 1997.
}

'Sylgard, Precision Instruments, Sarasota, Fla.

'Tomasic M. Effect of hypoxia on equine pulmonary arterial contractile response to norepinephrine (abstr). Vet Surg 1997;26:163.

\section{References}

1. Phillips TJ, Walmsley JP. Retrospective analysis of the results of 151 exploratory laparotomies in horses with gastrointestinal disease. Equine Vet J 1993;25:427-431.

2. Blikslager AT, Bowman KF, Levine JF, et al. Evaluation of factors associated with postoperative ileus in horses: 31 cases (1990-1992). J Am Vet Med Assoc 1994;205:1748-1752.

3. Lester GD. Modification of gastrointestinal motility in horses, in Proceedings. 18th Am Coll Vet Intern Med Forum 2000;153-155.

4. Gerring EEL, Hunt JM. Pathophysiology of equine postoperative ileus: effect of adrenergic blockade, parasympathetic stimulation and metoclopramide in an experimental model. Equine Vet J 1986;18:249-255.

5. Bishop AE, Hodson NP, Major JH, et al. The regulatory peptide system of the large bowel in equine grass sickness. Experientia 1984;40:801-806.

6. Goldin E, Karmeli E, Selinger Z, et al. Colonic substance P levels are increased in ulcerative colitis and decreased in chronic severe constipation. Dig Dis Sci 1989;34:754-757.

7. Singer II, Kawka DW, Scott S, et al. Expression of inducible nitric oxide synthase and nitrotyrosine in colonic epithelium in inflammatory bowel disease. Gastroenterology 1996;111:871-885.

8. Rodeberg DA, Chaet MS, Bass RC, et al. Nitric oxide: an overview. Am J Surg 1995;170:292-303.

9. Wirthlin DJ, Cullen JJ, Spates ST, et al. Gastrointestinal transit during endotoxemia: the role of nitric oxide. J Surg Res 1996;60:307-311.

10. Collins SM. The immunomodulation of enteric neuromuscular function: implications for motility and inflammatory disorders. Gastroenterology 1996;111:1683-1699.

11. Freeman DE, Cimprich RE, Richardson DW, et al. Early mucosal healing and chronic changes in pony jejunum after various types of strangulation obstruction. Am J Vet Res 1988;49:810-818.

12. Percy WH. In vitro techniques for the study of gastrointestinal motility. In: Gaginella TS, ed. Methods in gastrointestinal pharmacology. New York: CRC Press Inc, 1996;189-224.

13. Malone ED, Brown DR, Trent AM, et al. Influence of adrenergic and cholinergic mediators on the equine jejunum in vitro. Am J Vet Res 1996;57:884-890.

14. Bullitt E. Immediate-early genes and chronic pain. Am Pain Soc J 1994;3:53-55.

15. Burns GA, Cummings JF. Neuropeptide distributions in the colon, cecum, and jejunum of the horse. Anat Rec 1993;236:341-350.

16. Goyal RK, Hirano I. The enteric nervous system. N Engl J Med 1996;334:1106-1115.

17. Barthó L, Holzer P. Search for a physiological role of substance P in gastrointestinal motility. Neuroscience 1985;16:1-32.

18. Morteau O, More J, Pons L, et al. Platelet-activating factor and interleukin 1 are involved in colonic dysmotility in experimental colitis in rats. Gastroenterology 1993;104:47-56.

19. Burakoff R, Percy WH. Studies in vivo and in vitro on effects of $\mathrm{PGE}_{2}$ on colonic motility in rabbits. Am $\mathrm{J}$ Physiol 1992;262:G23-G29.

20. Kalff JC, Schraut WH, Simmons RL, et al. Surgical manipulation of the gut elicits an intestinal muscularis inflammatory response resulting in postsurgical ileus. Ann Surg 1998;5:652-663.

21. Muller MJ, Huizinga JD, Collins SM. Altered smooth muscle contraction and sodium pump activity in the inflamed rat intestine. Am J Physiol 1989;20:G570-G577.

22. Vermillion DL, Collins SM. Increased responsiveness of jejunal longitudinal muscle in Trichinella-infected rats. Am J Physiol 1988;254:G124-G129.

23. Cheung DW. Desensitization of the vascular contractile response to cumulative doses of $\alpha_{2}$-adrenoceptor agonists. Can J Physiol Pharmacol 1986;64:1343-1345.

24. Hirst GDS, McKirdy HC. Presynaptic inhibition at mammalian peripheral synapse? Nature 1974;250:430-431.

25. Barthó L, Holzer P, Lembeck F. Sympathetic control of substance $\mathrm{P}$ releasing enteric neurones in the guinea pig ileum. Neurosci Lett 1983;38:291-296. 